interaction such as that found in topological insulators. Because of their collective quantum properties, Majoranas also have unconventional (non-Abelian anyonic) statistics - they are neither bosons nor fermions - making them favourable for fault-tolerant quantum computing, and hence the search for Majorana fermions has become a priority in condensed-matter physics ${ }^{7}$. There have been several reports recently of evidence for Majoranas both in topological superconductors and in more conventional materials ${ }^{8,9}$.

The exotic properties predicted for topological insulators have prompted a gold-rush of research aimed at understanding and developing their potential. Initially it was thought that the topological-insulator class of materials was limited to only a few materials that have particular topological invariants in their electronic structure as well as a strong spin-orbit coupling. But Tanaka et al. ${ }^{1}$ in their work on $\mathrm{Pb}_{1-x} \mathrm{Sn}_{x} \mathrm{Te}$ alloys, and a simultaneously published study ${ }^{10}$ of $\mathrm{Pb}_{1-x} \mathrm{Sn}_{x} \mathrm{Se}$ in Nature Materials, have now demonstrated that crystal mirror symmetry also creates topologically protected surface states. And these topological crystalline insulators have a new feature: the Dirac spectrum can be gapped with applied strain, which gives additional capabilities.

It is becoming clear that topological materials with interesting properties are far more plentiful than originally thought, which bodes well for scientists being able to pinpoint those most suitable for practical application. For example, the PbSnTe system studied by Tanaka et al. is less prone to defect-induced doping, which is a serious issue in $\mathrm{Bi}_{2} \mathrm{Se}_{3}$ and related materials that are now most actively studied. The possibility of finding materials that could operate at room temperature and be compatible with existing technologies now seems more likely. And as it is the surface states that matter, it will also be important to home in on materials that have stable and controllable surface chemistry.

More topological materials are likely to be discovered as other potential classes are explored. These include materials that combine strong spin-orbit interaction with strong electron correlations (as in cuprate superconductors) or other types of order such as magnetism or superconductivity.
Interesting properties have already been predicted, such as three-dimensional Dirac spectra (Weyl states) and surface states with so-called Fermi $\operatorname{arcs}^{11}$; magnetic fluctuations imply a dynamical axion field, which may result in novel optical properties ${ }^{6}$. All this progress suggests that the future for topological materials is bright indeed.

H. Dennis Drew is in the Department of Physics, University of Maryland, College Park, Maryland 20742, USA.

e-mail:hdrew@umd.edu

\section{Reference}

1. Tanaka, Y. et al. Nature Phys. 8, 800-803 (2012).

2. Fu, L. Phys. Rev. Lett. 106, 106802 (2011).

3. Hsieh, T. H. et al. Nature Commun. 3, 982 (2012).

4. Kane, C. L. \& Mele, E. J. Phys. Rev. Lett. 98, 106803(2007).

. Appelbaum, I., Drew, H. D. \& Fuhrer, M. Appl. Phys. Lett. 98, 023103 (2011)

6. Li, R., Wang, J., Qi, X-L. \& Zhang, S-C. Nature Phys. 6, 284-288 (2010)

7. Sau, J. D., Lutchyn, R. M., Tewari, S. \& Das Sarma, S. Phys. Rev. Lett. 104, 040502 (2010)

8. Mourik, V. et al. Science 336, 1003-1007 (2012).

9. Williams, J. R. Phys. Rev. Lett. 109, 056803 (2012).

10. Dziawa, P. et al. Nature Mater. 11, http://dx.doi.org/10.1038/ nmat3449 (2012).

11. Pesin, D. \& Balents, L. Nature Phys. 6, 376-381 (2010)

\title{
COLLOIDAL CRYSTALS
}

\section{Perfect stranger}

Squeeze a foreign particle into a onedimensional crystal, and the system will readily rearrange itself to make room for the interloper. But try the same thing in two dimensions, and you will quickly find that the crystal is incapable of accommodating the change. Unless, that is, the planar surface of the two-dimensional crystal is curved - as William Irvine and colleagues have discovered, by imaging colloidal particles assembled on curved oil/glycerol interfaces (Nature Mater. http://doi.org/ jh3; 2012).

Wrapping a two-dimensional crystal onto a curved surface comes with its own set of difficulties: the change in geometry induces a frustration that in turn generates topological defects, such as dislocations and disclinations. These defects ease the frustration - effectively disrupting the crystalline order to relieve compressive and shear stresses. In the case of the colloidal crystal assembled by Irvine et al., the system relaxed into a pattern scarred by a set of pleated structures.

Using optical tweezers decoupled from their imaging system, the authors

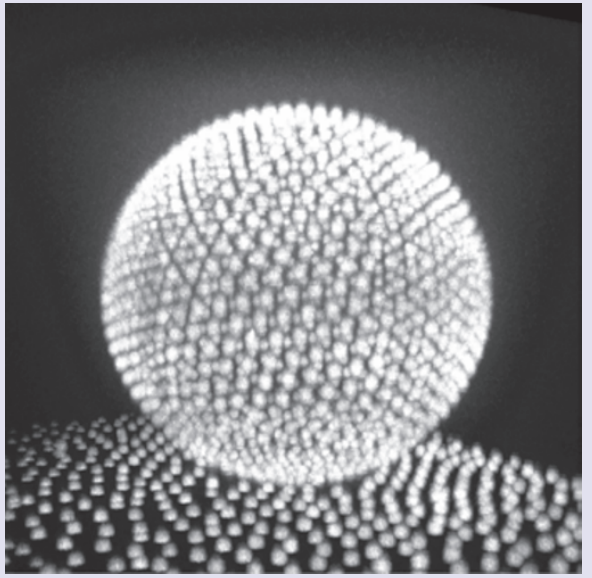

then introduced interstitial particles into their hexagonal colloidal crystal, and compared the ensuing dynamics on flat and curved surfaces. In both cases, the crystal accommodated the interstitials as expected, by forming pairs of particles with either five or seven neighbours, causing a spike in the local density field.

Ordinarily, on flat surfaces, these hexagonal lattice defects would be stable: they can diffuse through the lattice, but they leave dislocations bound. On curved surfaces, however, Irvine et al. found that the dislocations broke into pairs that also moved through the crystal, thereby flattening out the density spike. The actual particle introduced with the tweezers remained in the region to which it was added, but the entire region underwent a rotation as a result of the inclusion.

It turns out that the inherent topological defects brought on by frustration in curved space are key to the fractionalization of these dislocations. Irvine et al. found that the individual defect pairs migrated through the crystal until they were absorbed by defect boundaries already existing in the curved crystal. It may be possible to trigger this fractionalization in planar crystals under certain conditions, but the phenomenon arises quite naturally in curved space. Stress that is not relieved by the initial scarring effectively drives the self-healing process missing in flat crystals - recasting interstitials as order-restoring, rather than orderdisrupting, excitations.

ABIGAIL KLOPPER 